\title{
CARACTERIZAÇÃO DE FATORES QUE AFETAM A GERMINAÇÃO DE TECA (Tectona grandis): TEMPERATURA E ESCARIFICAÇÃO ${ }^{1}$
}

\author{
Rodrigo Barros Rocha², Abadio Hermes Vieira², Victor Mouzinho Spinelli³ e José Roberto Vieira²
}

RESUMO - A germinação lenta e irregular das sementes é uma limitação para a produção de mudas de teca (Tectona grandis). Embora métodos práticos estejam disponíveis poucos estudos quantificaram sistematicamente o efeito do aquecimento e da escarificação para a superação da dormência. O emprego de altas temperaturas pode ser realizado através de um coletor solar, aparelho de montagem simples que permite a captação da energia solar e transformação em energia calorífera. O objetivo deste trabalho foi avaliar a interação dos fatores "aquecimento" e "escarificação" na superação da dormência dos diásporos de teca avaliando o desempenho do coletor solar. Foi avaliado experimento em delineamento de fatorial completo com quatro níveis do fator "aquecimento" (A1 - solarizador, A2 - estufa a $80^{\circ} \mathrm{C}(12 \mathrm{~h}), \mathrm{A} 3$ - estufa a $80^{\circ} \mathrm{C}$ (4h/3dias), A4- sem aquecimento) e três níveis do fator "escarificação" (E1 - escarificação física, E2 - escarificação química e E3 - sem escarificação), totalizando 12 tratamentos com cinco repetições de 25 diásporos por parcela. O teste $\mathrm{F}$ a $1 \%$ de probabilidade indicou a ocorrência de interação significativa entre os fatores "aquecimento" e "escarificação", resultado da menor germinação dos diásporos que foram escarificados após aquecimento. A utilização de altas temperaturas na ausência de escarificação caracterizou os tratamentos de melhor desempenho para a superação da dormência. O menor desempenho associado aos maiores custos e riscos para manipulação desencorajaram o uso da escarificação química na superação da dormência. A alternância de temperaturas favoreceu a germinação, sendo que o coletor solar mostrou-se uma alternativa prática, viável e de menor custo para a superação da dormência em teca.

Palavras-chaves: quebra de dormência, produção de mudas, solarizador.

\section{CHARACTERIZATION OF FACTORS AFFECTING TEAK GERMINATION (Tectona grandis): TEMPERATURE AND SCARIFICATION}

\begin{abstract}
The slow and irregular seeds germination limits the production of teak seedlings (Tectona grandis). Although practical methods are available, few studies have systematically quantified the effect of heating and scarification to break the dormancy. High temperatures can be reached through a solar collector, simple assembly device that can capture and transform the solar energy into heat energy. This study aimed to evaluate the interaction of "heating" and "scarification" to overcome the dormancy of teak seeds, evaluating the solar collector performance. We evaluated experimentssome experiment in complete factorial design with four levels of the factor "heat" (A1 - solarized, A2 - greenhouse at $80^{\circ} \mathrm{C}(12 \mathrm{~h}), \mathrm{A} 3$ - greenhouse at $80^{\circ} \mathrm{C}(4 \mathrm{~h} / 3$ days), $\mathrm{A} 4$ - unheated) and three levels of the factor "scarification" (E1 - physical scarification, E2 - chemical scarification, and E3 and - without scarification), totaling 12 treatments with five replicates of 25 diaspores per plot. The F test at $1 \%$ of probability indicated the presence of significant interaction between factor heating and "scarification" and the lower germination result of the fruits that were scarified after heating. The use of high temperatures in the absence of scarification featured the best performing treatments to overcome dormancy. The lower performance associated with higher costs and risks for handling discouraged the chemical scarification use on dormancy break. Alternating temperatures enhanced the germination, and the solar collector proved to be a practical alternative, viable and less costly to overcome dormancy in teak
\end{abstract}

Keywords: Dormancy break, Seedling production and Solarization.

\footnotetext{
${ }^{1}$ Recebido em 22.07.2008 e aceito para publicação em 16.12.2011.

${ }^{2}$ Centro de Pesquisa Agroflorestal de Rondônia, CPAFRO, Brasil. E-mail: <rodrigo@cpafro.embrapa.br>.

${ }^{3}$ Universidade Federal de Viçosa, UFV, Brasil.
} 


\section{INTRODUÇÃO}

A Teca (Tectona grandis) é espécie nativa das florestas tropicais de monção do Sudeste asiático (Índia, Myanmar, Tailândia e Laos), que tem se destacado nos plantios na região amazônica pelo crescimento volumétrico e qualidade da madeira (FIGUEIREDO et al., 2005; BENTES-GAMA, 2005). A madeira da teca é valorizada no mercado internacional, apresentando preços mais elevados do que a madeira de mogno (Swietenia macrophylla King). A rusticidade, resistência a incêndios florestais e a qualidade da madeira são suas principais características (FIGUEIREDO, 2001).

Uma das principais limitações para a produção de mudas de teca é a germinação lenta e irregular das sementes inseridas em fruto de endocarpo e mesocarpo duros e de alta resistência. A germinação em campo apresenta taxa relativamente baixa de 25 a 35\% e desuniforme no período de 10 a 90 dias (KAOSA-ARD, 1986). Comercialmente, o que é chamado de semente, na realidade, trata-se do fruto, que pode conter até quatro sementes viáveis. Tratados como unidade de dispersão, os frutos são chamados de diásporos.

Os primeiros procedimentos para quebra de dormência de sementes de teca foram descritos há mais de 40 anos. Dabral (1967 citado por CALDEIRA e CALDEIRA, 2001) relatou germinação desuniforme de 50 a 79\% através da remoção manual do exocarpo e secagem ao sol por algumas semanas. Keiding (1985) e Kaosa-Ard (1986) sugeriram escarificar os frutos durante a noite e secar ao sol por uma a duas semanas. Ngulube (1989) observou 15\% de germinação com imersão em água por 48 h combinada com a remoção do exocarpo e alternância entre secagem e imersão por $12 \mathrm{~h}$. Lamprecht (1990) recomendou deixar os frutos em água corrente por $24 \mathrm{~h}$, secar ao sol e repetir o procedimento por duas semanas. Brasil (1992) sugeriu macerar os frutos em água e secar por 18 dias antes da semeadura. O baixo rendimento operacional e a menor praticidade são as principais limitações das metodologias conhecidas.

Resultados de pesquisas têm mostrado que altas temperaturas e a escarificação dos frutos são fatores determinantes para a superação da dormência (ALVES et al., 2007; REBELO, 2003; CALDEIRA e CALDEIRA, 2001; CALDEIRA e VIEIRA, 2001; CÁCERES FLORESTAL, 1997; BRASIL, 1992; PELUSO, 1995). Rebelo (2003) observou que o tratamento em estufa a $80^{\circ} \mathrm{C}$ por $12 \mathrm{~h}$ resultou em porcentagem de germinação de 78,5\% no vigésimo oitavo dia.
A influência positiva das altas temperaturas no processo de quebra de dormência pode ser obtida pela utilização de coletor solar, aparelho de montagem simples que permite a captação da energia solar e transformação em energia calorífera. Geralmente utilizado para a desinfestação de substratos, pode alcançar temperaturas tão elevadas quanto $80^{\circ} \mathrm{C}$ (GHINI, 2004; SOUZA 1994). A eficiência do solarizador irá depender, além de outros fatores, da natureza aditiva do efeito do calor recebido e do efeito benéfico da alternância de temperatura para a quebra de dormência, resultado da exposição por algumas horas diárias nas temperaturas mais elevadas (acima de $70{ }^{\circ} \mathrm{C}$ ).

O objetivo deste trabalho foi avaliar a interação dos fatores “aquecimento" e "escarificação” na quebra de dormência das sementes de teca, avaliando o desempenho de um coletor solar.

\section{MATERIAL E MÉTODOS}

\subsection{Coleta de sementes}

Os ensaios foram realizados no Laboratório de Análise de Sementes da Embrapa Rondônia, localizado em Porto Velho, no período de setembro a outubro de 2007. Os frutos foram colhidos na safra de 2007 em plantios da empresa SULMAP, localizada no Município de Pimenta Bueno, RO. Frutos menores do que $10 \mathrm{~mm}$ foram removidos por passagem em peneira e os frutos quebrados, retirados manualmente.

\subsection{Testes de germinação}

Os testes de germinação foram realizados conforme metodologia descrita pela Regras de Análise de Sementes - RAS (BRASIL, 1992), com duração de 35 dias em avaliações diárias após a primeira germinação. Os tratamentos de escarificação foram aplicados nos diásporos após os tratamentos de aquecimento. A escarificação física foi realizada utilizando escarificador elétrico, marca Weg, de 1.725 rotações por min, por 5 seg, com lixa número 60. A escarificação química foi realizada usando ácido sulfúrico P.A. (MERCK 95-98\%) por 15 min, seguido de lavagem abundante em água corrente.

Após a aplicação dos tratamentos, os diásporos foram semeados em "gerbox" contendo como substrato vermiculita esterilizada e umedecida. A reposição de água foi realizada semanalmente, conforme a necessidade. Posteriormente, o material foi armazenado em câmara 
de germinação (Tecnal-TE-401) por 35 dias, com controle de temperatura e luminosidade, alternando ciclos de $35^{\circ} \mathrm{C}$ e luminosidade por $12 \mathrm{~h}$ e $25^{\circ} \mathrm{C}$ no escuro por 12 h. Os diásporos foram dispostos em cinco linhas, a 1,0 cm uma da outra e com cinco frutos por linha, formando um quadrado. Foram considerados germinados os diásporos que apresentaram, até o trigésimo quinto dia, pelo menos uma plântula com cotilédones abertos e o primeiro par de folhas. Devido à dureza do mesocarpo dos frutos de teca e à ocorrência de 1-4 sementes viáveis por fruto, cada diásporo foi tratado como uma semente (KAOSA-ARD, 1986), como também considerado em outras espécies florestais (FIGLIOLIA et al., 1993).

As características avaliadas foram a porcentagem de germinação e o Índice de Velocidade de Germinação (IVG). Para interpretação dos dados, a porcentagem de germinação foi transformada em valor angular (utilizando o arco seno dos valores de porcentagem) e o índice de velocidade de germinação, estimado, como mostrado por Silva e Nakagawa (1995):

$$
I V G=\frac{G_{1}}{N_{1}}+\frac{G_{2}}{N_{2}}+\frac{G_{3}}{N_{3}}+\ldots \ldots+\frac{G_{n}}{N_{n}}
$$

em que IVG é o índice de velocidade de germinação, $G_{1}, G_{2}, G_{3}$ o número de plântulas germinadas, computadas na primeira, segunda até a última contagem; $N_{1}, N_{2}, N_{3}$ o número de dias da semeadura à primeira, segunda até a última contagem.

\subsection{Coletor solar}

Coletor solar de dimensões 1,5 m x 1,0 m x 0,3 m foi construído utilizando compensado naval, de acordo com as instruções (GUINI et al., 2004). O coletor foi instalado com exposição na face Norte com ângulo de inclinação de $18^{\circ}$, que corresponde à latitude local acrescida de $10^{\circ}$ (GUINI et al., 2004; MARTINS et al., 2003).

\subsection{Delineamentos experimentais}

Visando quantificar os efeitos e a interação entre os fatores “aquecimento” e “escarificação”, foi planejado um experimento em delineamento de fatorial completo com quatro níveis do fator "aquecimento" (A1- solarizador, A2- estufa a $80^{\circ} \mathrm{C}$ (12 h), A3- estufa a $80^{\circ} \mathrm{C}$ ( $4 \mathrm{~h} / 3$ dias), A4- sem aquecimento) e três níveis do fator “escarificação” (E1 - escarificação física, E2 - escarificação química e E3 - sem escarificação), resultando em 12 tratamentos com cinco repetições de 25 diásporos por parcela.
As análises foram realizadas segundo o modelo:

$Y_{i j k}=\mu+a_{i}+b_{j}+a b_{i j}+\varepsilon_{i j k}$

$Y_{i j k}$ : k-ésima observação no i-ésimo tratamento de “aquecimento", no j-ésimo tratamento de “escarificação”; $\mu$ : média geral do ensaio; $a_{i}$ :efeito do i-ésimo tratamento de "aquecimento"; $b_{j}$ : efeito do j-ésimo tratamento de "escarificação”; $a b_{i j}$ : efeito da interação entre os fatores "aquecimento" e de “escarificação”; $\varepsilon_{i j k}$ : erro aleatório. Todas as variáveis, exceto a média geral, foram consideradas como efeitos aleatórios.

Para quantificar o efeito do "tempo de exposição dos diásporos ao calor” no interior do coletor solar no período de um a 11 dias de exposição, foi planejado um segundo experimento em delineamento inteiramente casualizado com 12 tratamentos com cinco repetições de 25 diásporos por parcela.

As análises foram realizadas segundo o modelo:

$$
Y_{i j k}=\mu+a_{i}+\varepsilon_{i j k}
$$

$Y_{i j}$ : j-ésima observação no i-ésimo dia de exposição ao calor no coletor solar; $\mu$ : média geral do ensaio; $a_{i}$ :efeito do i-ésimo dia de exposição ao calor; e $\varepsilon_{i j}$ : erro aleatório. Todas as variáveis, exceto a média geral, foram consideradas como efeitos aleatórios.

\subsection{Obtenção de modelo para previsão de temperatura dentro do coletor}

Visando ao desenvolvimento de modelo de superfície de resposta para prever a resposta da variável dependente "temperatura interna do coletor solar", em função das variáveis independentes "temperatura externa”, “umidade” e "irradiação”, mensuradas em estação climática instalada na Embrapa Rondônia, em Porto Velho, RO, a temperatura dentro dos cilindros foi monitorada com um coletor de dados portátil, que registrou a temperatura a cada $10 \mathrm{~min}$. Visando verificar a importância dessas variáveis para a predição da temperatura dentro do aparelho, foi utilizado o método "stepwise” com probabilidade-limite para a variável entrar no modelo de 0,25 e permanecer no de 0,30 (YONEZAMA et al., 2000).

Foi ajustado modelo de regressão de acordo com o seguinte modelo:

$$
Y=\beta_{1} X_{1}+\beta_{2} X_{2}+\beta_{3} X_{3}
$$

Revista Árvore, Viçosa-MG, v.35, n.2, p.205-212, 2011 
em que é a temperatura interna no coletor solar, $\beta_{1}, \beta_{2}, \beta_{3}$ e são parâmetros do modelo de regressão, e $X_{1}, X_{2}, X_{3}$ e são variáveis independentes "temperatura externa”, “umidade” e “irradiação”.

\section{RESULTADOS E DISCUSSÃO}

O teste $\mathrm{F}$ a $1 \%$ de probabilidade indicou efeito significativo da interação entre os fatores "aquecimento" e “escarificação” (Tabela 1). Os valores dos coeficientes de variação foram compatíveis com os de outros trabalhos (CALDEIRA e VIEIRA, 2001; CALDEIRA e CALDEIRA, 2001). Em quatro dos 12 tratamentos avaliados, a diferença entre a maior e a menor repetição foi superior ao valor de tolerância máxima $\left(\mathrm{T}_{\max }=0,26\right)$, apresentado por Brasil (1992). Nesses casos, Brasil (1992) recomendou o descarte de uma das repetições, seguido de nova avaliação do valor da tolerância máxima. Nesses quatro tratamentos, a repetição mais discrepante foi descartada e substituída pela média das outras quatro repetições.

Entre outros mecanismos associados à germinação lenta e irregular da teca, a resistência do mesocarpo é um dos principais fatores limitantes da germinação, e o melhor procedimento para a quebra de dormência deve diminuir a resistência mecânica do fruto sem afetar a viabilidade das sementes. A ocorrência de interação significativa entre os fatores “aquecimento” e “escarificação” indica que um fator se comporta diferencialmente na presença do outro e não devem ser interpretados isoladamente (Tabela 1).

De maneira geral, observa-se menor taxa de germinação nos tratamentos em que os frutos foram escarificados (Tabela 2). Foi observada menor taxa de germinação nas sementes que passaram pela escarificação
(E1 e E2) após a aplicação dos tratamentos, (A1) e (A3), que tem em comum a alternância de temperaturas. A escarificação de frutos fragilizados pela ação do calor pode reduzir a viabilidade das sementes, associado à maior taxa de ruptura dos frutos observada dentro do escarificador dos tratamentos que foram aquecidos. O desempenho do tratamento escarificado (E1A4) não diferiu do tratamento-controle (E3A4), pelo teste de Tukey a 1\% de probabilidade. Resultados semelhantes foram obtidos por Rebelo (2003), Souza et al. (2007) e Vieira et al. (2008).

As sementes que passaram pela escarificação química apresentaram redução significativa na sua taxa de germinação e menor desenvolvimento das plântulas (Tabela 2). Nesse tratamento, observou-se incidência de fungos sobre a maior parte dos diásporos, o que pode estar associado aos açúcares produzidos durante a hidrólise ácida da celulose com o ácido sulfúrico. Resultados semelhantes foram encontrados por Martinelli-Seneme et al. (2006). Além do menor desempenho, o maior custo e o maior risco de manipulação desencorajam a utilização da escarificação ácida para a superação da dormência da teca. Rebelo (2003) observou que a média da escarificação ácida não diferiu da do controle.

A interpretação individual dos fatores mostrou que "aquecimento" foi o fator mais eficiente para a quebra de dormência. Na ausência da escarificação, os tratamentos (A1E3), (A2E3) e (A3E3) não diferem pelo teste de Tukey a $1 \%$ de probabilidade, no trigésimo quinto dia, indicando que o coletor solar pode substituir a estufa, e a alternância das temperaturas não prejudicou a quebra de dormência (Tabela 2). A interpretação semanal

Tabela 1 - Resumo da análise de variância individual que testa a igualdade das médias das características de germinação. Table 1 - Abstract of individual ANOVA that tests the averages equality of germination features.

\begin{tabular}{ccccc}
\hline ANOVA & G.L. & $\mathrm{G}_{\%}$ & Arcsen $\left(\mathrm{G}_{\%}\right)$ & IVE \\
\hline AQC & & & $\mathrm{F}$ & $1,16^{\mathrm{ns}}$ \\
ESC & 3 & $0,54^{\mathrm{ns}}$ & $0,58^{\mathrm{ns}}$ & $16,75^{* *}$ \\
AQC x ESC & 2 & $10,58^{* *}$ & $10,54^{* *}$ & $5,76^{* *}$ \\
Resíduo & 6 & $9,22^{* *}$ & $8,87^{* *}$ & \\
Total & 48 & & & 0,93 \\
\hline Média Geral & 59 & & 0,77 & 19,06 \\
CV & & 0,49 & 10,44 & \\
\hline
\end{tabular}

AQC: aquecimento, ESC: escarificação, AQC x ESC: Interação entre os fatores, aquecimento e escarificação, G.L.: graus de liberdade, G\%: percentual de germinação, Arcsen(G\%): arco-seno dos valores observados do percentual de germinação, IVE: índice de velocidade de germinação, CV: coeficiente de variação.

Revista Árvore, Viçosa-MG, v.35, n.2, p.205-212, 2011 
Tabela 2 - Valores médios da germinação de sementes estimados em fatorial completo com doze tratamentos. As letras indicam a classificação das médias obtida pelo teste de Tukey a 1\% de probabilidade. Os testes de média foram estimados a partir dos dados transformados em valor angular utilizando o arco seno dos valores da porcentagem de germinação.

Table 2 - Average seeds germination values obtained in a complete factorial design with twelve treatments. The letters indicate the average rating obtained by Tukey test at $1 \%$ of probability. The mean tests were estimated from the transformed data value using the arcsine values of the germination percentage

\begin{tabular}{|c|c|c|c|c|}
\hline \multicolumn{5}{|c|}{ Porcentagem de germinação aos 35 dias } \\
\hline Tratamentos & $\begin{array}{l}\text { Solarizador } \\
\text { (A1) }\end{array}$ & $\begin{array}{l}\text { Estufa } 80^{\circ} \mathrm{C} \\
(12 \mathrm{~h})(\mathrm{A} 2)\end{array}$ & $\begin{array}{c}\text { Estufa } 80^{\circ} \mathrm{C} \\
\text { (4h/dia) (A3) }\end{array}$ & $\begin{array}{l}\text { Sem aquec. } \\
\text { (A4) }\end{array}$ \\
\hline Escarificação física (E1) & $0,41 \mathrm{Ab}$ & $0,54 \mathrm{Aa}$ & $0,49 \mathrm{Ab}$ & $0,55 \mathrm{Aa}$ \\
\hline Escarificação química (E2) & $0,38 \mathrm{Ab}$ & $0,34 \mathrm{Ab}$ & $0,28 \mathrm{Ac}$ & $0,28 \mathrm{Ab}$ \\
\hline Sem escarific. (E3) & $0,80 \mathrm{Aa}$ & $0,66 \mathrm{Aa}$ & $0,70 \mathrm{Aa}$ & $0,46 \mathrm{Ba}$ \\
\hline \multicolumn{5}{|c|}{ Velocidade de germinação avaliada até o $35^{\circ}$ dia } \\
\hline Tratamentos & $\begin{array}{c}\text { Solarizador } \\
\text { (A1) }\end{array}$ & $\begin{array}{l}\text { Estufa } 80^{\circ} \mathrm{C} \\
(12 \mathrm{~h})(\mathrm{A} 2)\end{array}$ & $\begin{array}{c}\text { Estufa } 80^{\circ} \mathrm{C} \\
\text { (4h/dia) (A3) }\end{array}$ & $\begin{array}{c}\text { Sem aquec. } \\
\text { (A4) }\end{array}$ \\
\hline Escarificação física (E1) & $0,91 \mathrm{Ab}$ & $1,13 \mathrm{Aa}$ & $1,02 \mathrm{Aa}$ & $1,03 \mathrm{Aa}$ \\
\hline Escarificação química (E2) & $0,64 \mathrm{Ab}$ & $0,52 \mathrm{Ab}$ & $0,43 \mathrm{Ab}$ & $0,43 \mathrm{Ab}$ \\
\hline Sem escarific. (E3) & $1,60 \mathrm{Aa}$ & $1,30 \mathrm{Aa}$ & $1,35 \mathrm{Aa}$ & $0,85 \mathrm{Ba}$ \\
\hline
\end{tabular}

Médias seguidas pelas mesmas letras maiúsculas na HORIZONTAL não diferem estatisticamente entre si. Médias seguidas pelas mesmas letras minúsculas na VERTICAL não diferem estatisticamente entre si.

mostra a tendência de superioridade no desempenho dos tratamentos que utilizam a alternância de temperaturas (Figura 1). O efeito benéfico dessa alternância pode estar relacionado tanto com resposta fisiológica das sementes, quanto como redução na resistência mecânica do mesocarpo que as reveste.

O efeito do tempo de exposição ao calor dentro do coletor solar indicou que pelo menos dois dias foram necessários para o aumento na taxa de germinação (Figura 2). Não foi observada redução no percentual de germinação pela exposição de até 11 dias no coletor.

Visando prever a oscilação de temperatura dentro do solarizador, foi desenvolvido um modelo em função das variáveis “temperatura externa”, “umidade” e "radiação incidente”. O método “stepwise” de seleção de variáveis indicou que as três características foram importantes para a predição da variável dependente. A análise de resíduos mostrou tendência de aumento do erro com o valor das estimativas, limitação essa, associada à natureza da variável resposta de aumento acentuado em curto período de tempo (Figura 2). O coeficiente de determinação ajustado indicou que a maior parte da variação total da variável dependente é explicada pelo modelo $\left(R_{a}^{2}=0,94\right)$, apresentado a seguir:

$T_{\text {int }\left({ }^{\circ} \mathrm{C}\right)}=1,02277 \cdot T_{\operatorname{ext}\left({ }^{\circ} \mathrm{C}\right)}-0,02822 \cdot U_{(\%)}+0,01644 \mathrm{Rad}_{\left.(\mathrm{KJm})^{2}\right)}$

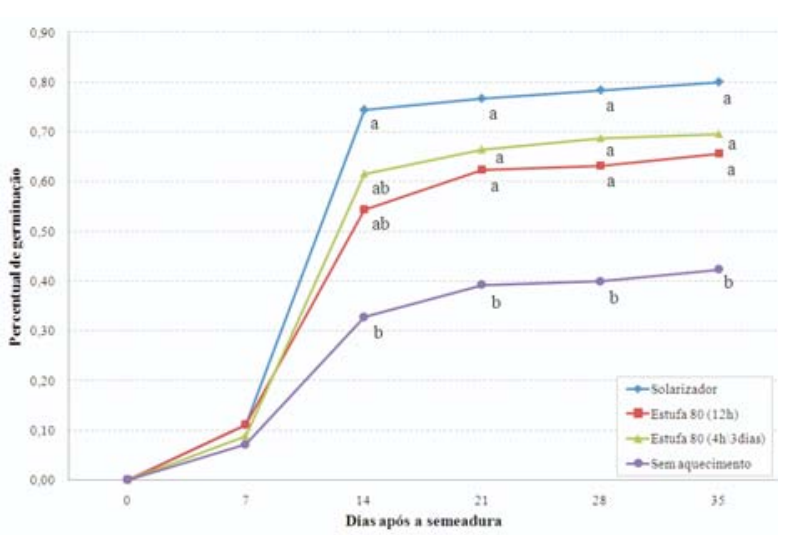

Figura 1 - Porcentagem de germinação ao longo das semanas dos tratamentos não escarificados (A1E3 solarizador, A2E3 - estufa a $80^{\circ} \mathrm{C}(12 \mathrm{~h}), \mathrm{A} 3 \mathrm{E} 3$ - estufa a $80^{\circ} \mathrm{C}$ (4h/3dias), A4E3 - sem aquecimento). As letras indicam a classificação das médias obtida pelo teste de Tukey a $1 \%$ de probabilidade, estimadas a partir dos dados transformados em valor angular utilizando o arco seno dos valores de porcentagem.

Figure 1 - Germination percentage over the weeks of not scarified treatments (A1E3 - solar collector, A2E3 - greenhouse at $80^{\circ} \mathrm{C}(12 \mathrm{~h}), \mathrm{A3E} 3$ - greenhouse at $80^{\circ} \mathrm{C}$ ( $4 \mathrm{~h} / 3$ days), A4E3 - no heating). The letters indicate the average rating obtained by Tukey test at $1 \%$ of probability estimated from the transformed data value using the arcsine values of the germination percentage

Revista Árvore, Viçosa-MG, v.35, n.2, p.205-212, 2011 


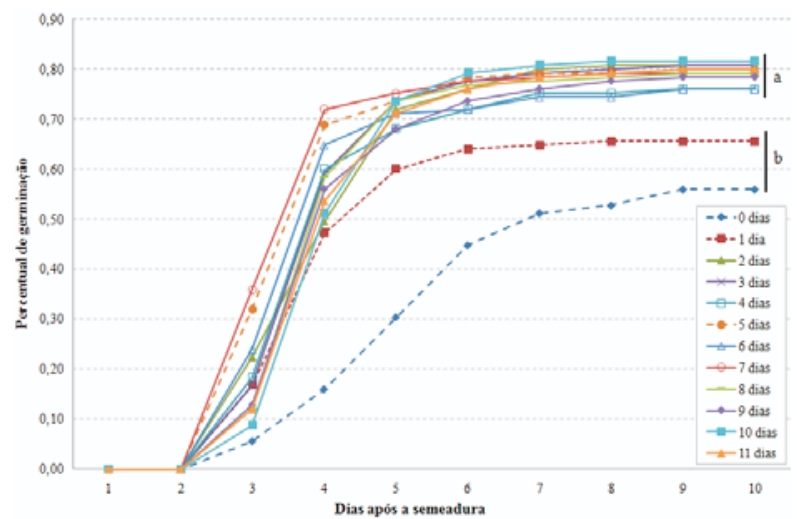

Figura 2 - Distribuição dos valores de germinação percentual avaliada diariamente. Os números de 0 a 11 correspondem ao número de dias de exposição ao calor no interior do coletor solar. As linhas identificadas pela mesma letra não diferem no porcentual de germinação segundo o teste de Tukey a $1 \%$ de probabilidade no vigésimo sétimo dia.

Figure 2 - Distribution of the germination values percentage assessed daily. The numbers from 0 to 11 represent the days quantity of heat exposure inside the solar collector. The lines identified by the same letter do not differ in the germination percentage, according to the Tukey test at $1 \%$ of probability at the twenty-seventh day.

em que temperatura interna do coletor em ${ }^{\circ} \mathrm{C}$, temperatura externa em ${ }^{\circ} \mathrm{C}$, umidade percentuammn vl e irradiação recebida em $\mathrm{KJm}^{2}$.

Tendo em vista que o investimento para a construção do solarizador é relativamente baixo, considerou-se a mudança de temperatura dentro do coletor causada pelas oscilações climáticas como sua principal limitação. O período de julho a setembro, em que a pluviosidade diminui sensivelmente no Estado de Rondônia, é especialmente propício para a utilização desse equipamento. Observou-se que em dia de irradiação plena o aparelho proporciona aproximadamente $4 \mathrm{~h}$ de temperatura entre 70 e $80^{\circ} \mathrm{C}$ e $3 \mathrm{~h}$ entre 60 e $70^{\circ} \mathrm{C}$, podendo atingir temperatura superior a $80^{\circ} \mathrm{C}$ (Figura 3 ).

O aumento da uniformidade e taxa de germinação foi o principal benefício do uso do calor seco com alternância de temperaturas para a superação da dormência. Em comparação com metodologias tradicionais, esse método tem o potencial para proporcionar redução nos custos e aumento da uniformidade nas mudas, impactando positivamente sobre a homogeneidade do plantio.

Revista Árvore, Viçosa-MG, v.35, n.2, p.205-212, 2011

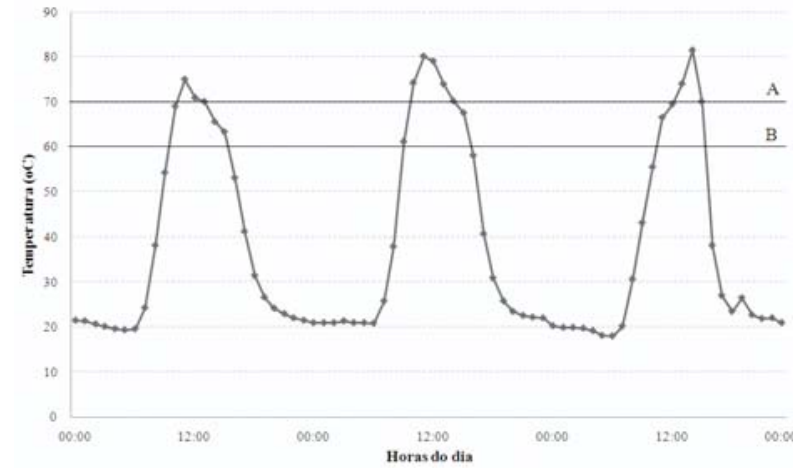

Figura 3 - Dispersão dos valores de temperatura registrada a cada 10 minutos no interior do coletor solar, no período de 3 a 5 de setembro de 2007 . A representa o período de maior aquecimento do aparelho de $70^{\circ}$ a $80^{\circ} \mathrm{C}$ e $\mathrm{B}$ - a período aquecimento intermediário entre $60^{\circ}$ a $70^{\circ} \mathrm{C}$.

Figure 3-Dispersion of the temperature values recorded every 10 minutes inside the solar collector from September 3 to 5, 2007. A - represents the device period greatest warming, 70 to $80^{\circ} \mathrm{C}$, and $\mathrm{B}$ the intermediate heating period between 60 to $70^{\circ} \mathrm{C}$.

\section{CONCLUSÕES}

Diante dos resultados, chegou-se às seguintes conclusões:

i) A utilização de altas temperaturas na ausência de escarificação caracterizou os tratamentos de melhor desempenho na superação da dormência da teca.

ii) O menor desempenho, associado aos maiores custos e riscos para manipulação, tem desencorajado a utilização da escarificação química para superação da dormência da teca.

iii) Pelo menos dois dias de exposição ao calor do coletor solar foram necessários para o aumento da taxa de germinação.

iv) A alternância de temperaturas favoreceu a germinação, e o coletor solar mostrou-se alternativa prática, viável e de menor custo para a quebra de dormência de sementes da teca.

\section{REFERÊNCIAS}

ALVES E. U. et al. Superação da dormência em sementes de Caesalpinia pyramidalis Tul.

Revista Árvore, v.31, n.3, p.405-415, 2007. 
BENTES-GAMA, M. Orientações para pesquisa florestal em Rondônia. Porto Velho: Embrapa Rondônia Porto Velho, 2005. 4p. (Comunicado Técnico, 273).

BRASIL. Ministério da Agricultura e Reforma Agrária. Regras para análise de sementes. Brasília: SNDA/DNDV/CLAV, 1992. 365p.

CÁCERES FLORESTAL. Manual do reflorestamento da Teca. Cáceres: 1997. 30p.

CALDEIRA, S. F., CALDEIRA, S. A. F. Efeito da imersão prévia em água e períodos de aquecimento, na viabilidade de sementes de Теса (Tectona grandis L.f.) Revista Agricultura Tropical, v.5, n.1, p.45-55, 2001.

CALDEIRA, S. F.; VIEIRA, E. P. Emergência de plântulas de teca, Tectona grandis L.f., com tratamentos pré-germinativos, em diferentes substratos. Revista Agricultura Tropical, v.5, n.1, p.81-90, 2001.

DABRAL, S. L. Extraction of teak seeds from fruits, their storage and germination. Indian Forester, v.102, n.10, p.650-658, 1967.

FIGUEIREDO, E. O.; OLIVEIRA, A. D.; SCOLFORO, J. R. S. Análise econômica de povoamentos não desbastados de Tectona grandis L.f. na microrregião do baixo rio Acre. Cerne, v.11, n.4, p.342-353, 2005.

FIGUEIREDO, E. O. Reflorestamento com Teca (Tectona grandis L.f.) no Estado do Acre. Rio Branco: Embrapa Acre, 2001. 28p. (Documentos, 65).

FIGLIOLIA, M. B.; OLIVEIRA, E. C.; PIÑARODRIGUES, F. C. M. Análise de sementes. In: AGUIAR, I. B.; PIÑA-RODRIGUES, F. C. M.; FIGLIOLIA, M. B (Coords.). Sementes florestais tropicais. Brasília: Abrates, 1993. p.137-174.

GHINI, R. Coletor solar para desinfestação de substratos para produção de mudas sadias. Jaguariúna: Embrapa Meio Ambiente, 2004. 28p. (Circular Técnica, 4).

KAOSA-ARD, A. Teak (Tectona grandis Linn. f.) nursery techniques with special reference to Thailand. Denmark: Danida Forest Seed Centre, 1986. 42p. (Seed Leaflet, 4A)
KEIDING, H. Teak, Tectona grandis Linn. f. Humiebaeck. Denmark: Danida Forest Seed Centre, 1985. 21p. (Seed leaflet, 4A)

LAMPRECHT, H. Silvicultura nos trópicos: ecossistemas florestais e respectivas espécies arbóreas - possibilidades e métodos de aproveitamento sustentado. Rossford: TZ-verl. Ges., 1990. 343p.

MARTINELLI-SENEME, A. et al. Germinação e sanidade de sementes de Bauhinia variegata. Revista Árvore, v.30, n.5, p.719-724, 2006.

MARTINS, M. V. V. et al. Erradicação de escleródios de Sclerotium rolfsii em substratos tratados em coletores solares em Campos dos Goytacazes - RJ. Revista Brasileira de Fruticultura, v.25, n.3, p.421-424, 2003.

NGULUBE, R. M. Seed germination, seedling growth and biomass production of eight central American multipurpose trees under nursery condition in Zomba, Malawi. Forest Ecology and Management, v.27, n.1, p.21-27, 1989.

PELUSO, E. B. Estudo sobre tratamentos pré-germinativos dos frutos e substratos mais adequados à germinação para produção de mudas de Teca (Tectona grandis L.f.) 1995. Monografia (Graduação Engenharia Florestal) Faculdade de Engenharia Florestal, Universidade Federal de Mato Grosso, Cuiabá, 1995.

REBELO, A. M. Estudo de aspectos morfológicos e de crescimento quantitativo de Tectona grandis (L.f.) no estado de Rondônia. Porto Velho: Fundação Universidade Federal de Rondônia, 2003. 56p.

SILVA, J. B. C.; NAKAGAWA, J. Estudo de fórmulas para cálculos de velocidade de germinação. Informativo ABRATES, v.5, n.1, p.62-73, 1995.

SOUZA, N. L. Solarização do solo. Summa Phytopathologica, v.20, n.1, p.3-15, 1994.

SOUZA, E. B. et al. Germinação de sementes de Adenanthera pavonina L. em função de diferentes temperaturas e substratos. Revista Árvore, v.31, n.3, p.437-443, 2007.

Revista Árvore, Viçosa-MG, v.35, n.2, p.205-212, 2011 
VIEIRA, A. H.; ROCHA, R. B.; REBELO, A. M. Avaliação de métodos para a superação de dormência de diásporos de teca (Tectona grandis L.f.). Revista Floresta, v.39, n.2, p.273-278, 2009.
YONEZAMA, K.; ISHII, T.; YANO, K. Definition of the Probability Efficiency Index for Optimization of the Stepwise Yield Selection Procedures of Plant Varieties. Biometrics, n.56, p.1213-1217, 2000. 